\title{
A Realistic Computed Tomography Simulator for Small Motion Analysis of Cerebral Aneurysms*
}

\author{
Shima Sepehri, Karim Zouaoui and Jean-Philippe Thiran, Senior Member, IEEE
}

\begin{abstract}
This paper describes a realistic simulator for the Computed Tomography (CT) scan process for motion analysis. In fact, we are currently developing a new framework to find small motion from the CT scan. In order to prove the fidelity of this framework, or potentially any other algorithm, we present in this paper a simulator to simulate the whole $\mathrm{CT}$ acquisition process with a priori known parameters. In other words, it is a digital phantom for the motion analysis that can be used to compare the results of any related algorithm with the groundtruth realistic analytical model. Such a simulator can be used by the community to test different algorithms in the biomedical imaging domain. The most important features of this simulator are its different considerations to simulate the best the real acquisition process and its generality.
\end{abstract}

\section{INTRODUCTION}

Computed Tomography (CT) scan is one of the most accurate imaging techniques that is used ubiquitously in medical diagnosis.

There exist a few studies about the CT simulation, such as the software provided in [1], or the descriptive, nonanalytical literature works of [2] and [3]. However, none of them -even [1]- provide the capability of analytical motion input which is required as an accurate digital ground-truth for critical diagnostic applications, as will be explained in the following. Therefore, to the best of the authors' knowledge, there is a lack of scientific work in this domain, to the point that we could not find any similar previous results to which we can compare ours.

In this paper we present a piece of work which is part of a larger project aiming to help the diagnostic and treatment of a severe pathology called intracranial aneurysm (IA) which is the pathological enlargement of a brain artery.

IA is a very dangerous and widespread problem. Fortunately, by the increasing use of medical imaging in different diagnoses, un-ruptured aneurysms are nowadays detected fortuitously while checking for other problems and may be treated in one of three ways: preventive surgery,

\footnotetext{
*Research supported by the European Commission FP7 framework Program under grant FP7-ICT-2009-6- 269966 (Thrombus).

Sh. Sepehri is with the Signal Processing Laboratory (LTS5), Ecole Polytechnique Fédérale de Lausanne (EPFL), EPFL STI IEL LTS5, Station 11, 1015 Lausanne, Switzerland (corresponding author, phone: +41 21693 56 58; fax: +412169376 00; e-mail: shima.sepehri@epfl.ch).

$\mathrm{K}$. Zouaoui is with the Laboratoire de Médecine Expérimentale (ULB 222 Unit), Université Libre de Bruxelles, CHU de Charleroi, 6110 Montigny-le-Tilleul, Belgium (email : karim.zouaoui@chu-charleroi.be)

$\mathrm{J}-\mathrm{Ph}$. Thiran is with the Signal Processing Laboratory (LTS5), Ecole Polytechnique Fédérale de Lausanne (EPFL), and the Department of Radiology, University Hospital Center (CHUV) and University of Lausanne (UNIL), EPFL STI IEL LTS5, Station 11, 1015 Lausanne, Switzerland (email: jp.thiran@epfl.ch).
}

neuro-radiological intervention or careful observation. At the moment, treatment depends mainly on the size and location of the aneurysm. According to studies [4], and considering the risks involved in surgery, careful observation is preferred for small aneurysms. However, a study in 2004 of 280 patients with ruptured aneurysms showed that a large proportion had small or very small aneurysms [5]. This means that at the moment there is no reliable means of predicting the rupture. Since observations during surgery shows that the aneurysms pulse ([4]-[8]), medical researchers are keen to study the relationship between the pulsatility and the rupture ([9]-[11]). However, there is no existing reliable method to detect this motion.

In the framework of the European project Thrombus (ref. FP7-ICT-2009-6- 269966), we are currently developing a new methodology to detect and quantify small motions of the aneurysm wall from dynamic CT data. To avoid resolution loss and noise generated by $\mathrm{CT}$ image reconstruction, our method exploits directly the sinograms ${ }^{1}$ generated by the CT data acquisition ([12]-[14]). This methodology is still under development, but in order to validate it, we have developed a complete numerical simulator, which can simulate small motion of an object (in our case of the aneurysm wall) and generate realistic sinogram data, as they would be generated by a real CT scanner scanning the corresponding real object. In particular, we can generate synthetic model of the organ and add known but realistic motion model to it and compare exactly the results of different motion quantification techniques with the ground-truth motion. In other words, this simulator provides the research community with an analytical phantom with small motion and the corresponding CT sinograms for medical image processing. This simulator can play an important role in the analytical and exact validation process, which is a necessary component in order to make algorithms useful for critical applications.

\section{II.PROCEDURE FOR CT SIMULATION}

\section{A. Input Parameters \& Simulator}

Firstly, the simulator simulates the organ moving volume. In this case, we simulated a volume around the IA by considering the aneurysm as a circle/sphere with realistic gray level intensity of the IA and its background (for example 200 Hounsfield Units (HU) and $100 \mathrm{HU}$, respectively). Of course, any other reference model can be used.

In order to cope with the limited resolution of CT images, the model has to be of high resolution as compared to the final reconstructed image. In this paper, for example, we use

\footnotetext{
${ }^{1}$ The sinogram is the raw data registered by a CT scanner. It is the footprint of the X-ray passing through the object. This concept will be explained in the next part.
} 
a 2D model image of size 1000 pixels by 1000 pixels to simulate a final image of resolution 100 by 100 pixels.

On that high resolution image, different motion models can be applied to arbitrary places of the volume. As suggested in [15] and because of the flexibility of such models, we used a cubic B-Spline as the motion model. The cubic B-Spline is defined as [16]

$$
\beta^{3}(x)= \begin{cases}\frac{2}{3}-\frac{1}{2}|x|^{2}(2-|x|) & 0 \leq|x|<1 \\ \frac{1}{6}-(2-|x|)^{3} & 1 \leq|x|<2 \\ 0 & \text { otherwise }\end{cases}
$$

Therefore, we can model image deformation due to motion by a cubic B-Spline in each of the two (or three) dimensions. It is a smooth shape, like those shown in fig. 1, with a width of 2. By multiplication of the two or three BSplines, we can apply smooth deformations similar to the real ones to IA or eventually any other organs.

Then we used a backward model to find the intensity of each pixel in the deformed image using the following equation, as in [15]

$$
\mathbf{q}=\mathbf{p}+\sum_{c} \boldsymbol{\beta}\left(\frac{\mathbf{p}-\mathbf{p}_{\mathbf{c}}}{\delta_{\mathbf{c}}}\right) \omega_{c}
$$

In equation (2), $\mathrm{p}$ and $\mathrm{q}$ are the coordinates of the points on the deformed and original images, respectively. $p_{c}$ are the control points of the B-Splines, which are the points to which we applied the B-Splines. $\delta_{c}$ define the width of the Splines and $\omega_{c}$ are the parameter of the splines at each control point, which practically define their amplitudes.

The backward model of equation (2) and the two BSplines multiplied to get a deformation are depicted in fig. 1, where only one control point is used, located at point $(543,160)$.

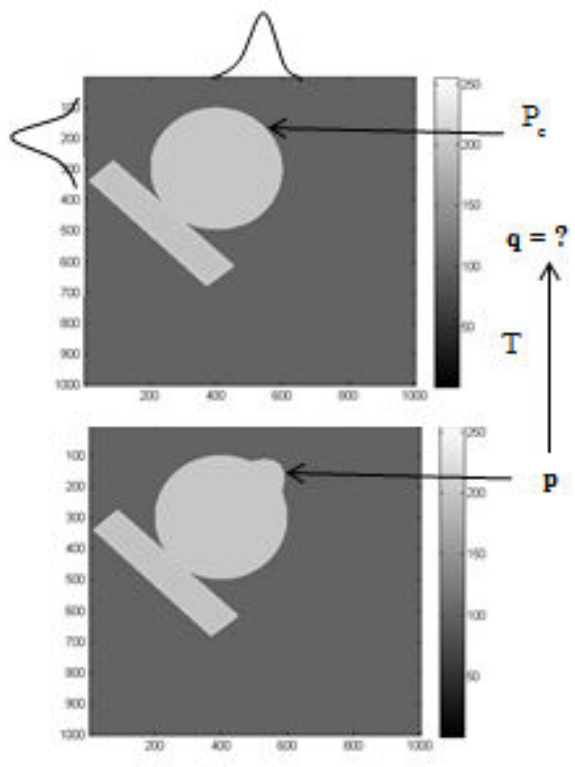

Figure 1. The backward model of the motion and the two 1D cubic BSplines used to create the motion: (a) original model, (b) deformed model using B-Splines deformation.
This image shows that for each pixel (p) on the deformed image we look for the corresponding pixel $(\mathbf{q})$ on the original image, using the equation (2). The gray level of $\mathbf{p}$ in the deformed image is the gray level of $\mathbf{q}$ in the original image.

Summarizing the previously-mentioned steps, we first create a high resolution image as a realistic estimation of the continuous reality. Then an arbitrary motion data can be applied to this image. To simulate aneurysm wall motion due to patient's heartbeat, we modulate the amplitude of the cubic B-Splines through time, for example, as a sinusoid, generating a temporal series of images with varying deformation (see fig. 2).

Indeed, according to [15], for the intracranial aneurysm, a B-Spline is a flexible and realistic estimation of the wall motion. Therefore, we applied different B-Spline deformations for different time instances. The amplitude of the B-Spline is changed with a sinusoid model with its peaks corresponding to the ECG. However, the interest of this simulator is that we can easily replace this amplitude model with an arbitrary periodic signal. In the same way, we can replace the B-Spline with affine or other motion model.
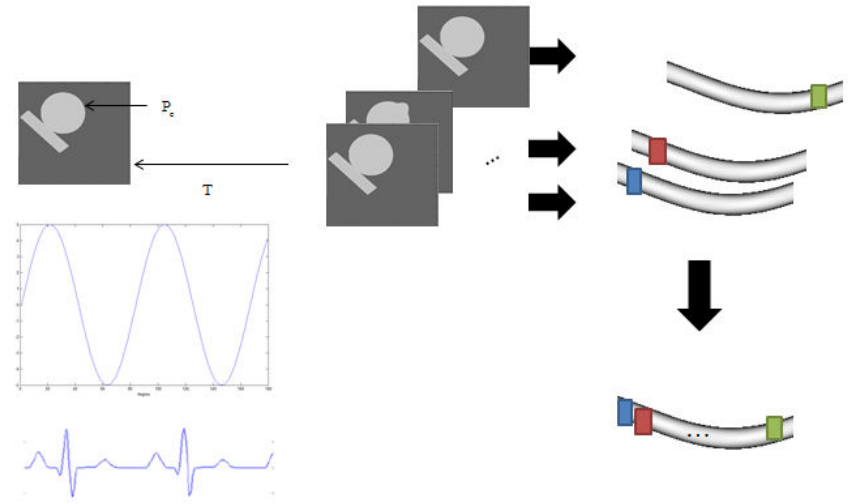

Figure 2. The sinogram of a moving object is made by putting together the projections of the object at different motion states

In the above figure, on the left hand side, we see the highresolution image deformed according to the previouslyexplained modulated B-Spline. The right hand side shows the sinograms which will be explained in the following subsection.

Now that we have a realistic temporal series of highresolution images simulating aneurysm wall motion, we have to generate the corresponding sinograms. Therefore, the simulator also inputs the velocity of the CT scan machine in degree per second, the total number of full turns $\left(360^{\circ}\right)$ that the machine scans and the time intervals in which the machine registers data.

\section{B. Outputs}

Using these inputs, the simulator can generate the sinogram of the moving object.

We recall that depending on the medical image modality, different raw data are registered by the machine. For example, for CT imaging these data are footprints of the attenuation of the X-ray while passing the object, called a sinogram. We give first in fig 3 a graphical explanation about the sinogram formation Moreover, in this picture we 
can see how the motion is reflected in the sinogram.

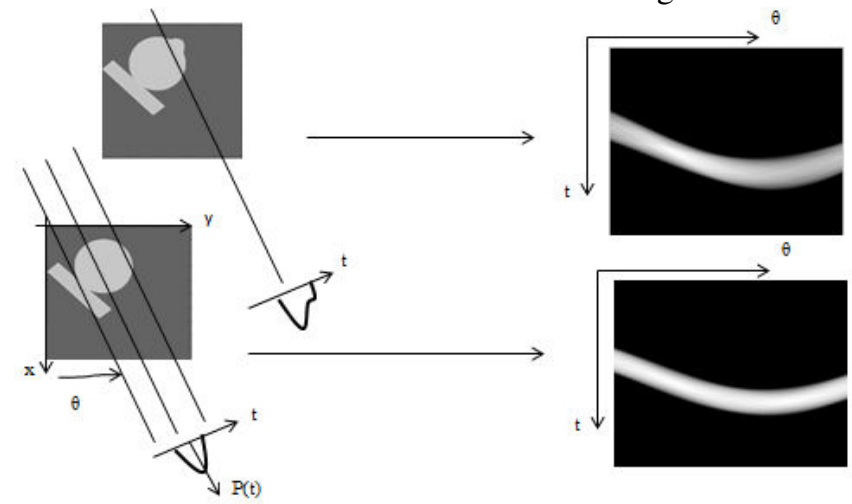

Figure 3. Illustration of a sinogram and the reflection of the motion of the object in this data.

The axes $t$ shows the sensors that register the attenuation of the X-ray. Then the width of this footprint is reported in the same axis in the $2 \mathrm{D}$ diagram on the right, called a sinogram. $\theta$ shows the angle while the set of the source and detectors is rotated around the object, as in a CT scan.

Another interesting feature is that we can set the number of sensors/captors along the t-axis in fig. 3, as a parameter of the simulation, to better simulate the reality of each scan and the source of the error of limited resolution.

For more information on sinograms, the interested reader is referred to [17].

The motion of the organ results in this kind of raw data (sinogram) which is composed of different parts, each part corresponds to a motion state regarding the step size of the registration. This was also taken into account in the simulation of these data.

To explain, it should be mentioned that each column of the sinogram is generated as follows: the time point of that column is considered. Then the high-resolution image corresponding to that time point is used, and projected in the direction corresponding to the rotation angle of the CT scanner at that time point. Finally, the projected signal is down sampled to reach the special resolution corresponding to the number of detectors of the simulated CT scanner. This process is repeated for each time point, generating the different columns of the desired sinogram, as shown in fig. 2.

On the right hand side of this figure, we can see how the corresponding parts of sinograms are put together to make the sinogram of a moving object. During the horizontal axis of a sinogram ( $\theta$ in fig. 3 ), the moving object is at a different motion state for each $\theta$. Therefore, to simulate the sinogram registered by a CT machine from a moving object, we put together the corresponding parts of the sinograms at different motion states, for the corresponding angles of rotation $(\theta)$. Finally, the simulator adds random noise to the sinogram as the real machine has errors in registering the data by the sensors.

With this result, we can for instance simulate the gating process used in dynamic EEG-gated CT imaging, i.e. produce the gated sinogram. Since the object is moving, with a periodic rhythm, gated CT image reconstruction puts together different parts of the sinogram which correspond to the same motion state regarding the ECG signal. Hence, we have the exact sinogram segments for each motion state. This is typically done by a windowing of the sinogram around the times corresponding to the same motion state, for example with a window but any other window can be easily replaced. This windowing has another important effect: it brings about a kind of smoothing in time ( $4^{\text {th }}$ dimension). It means that by using the window, we can relate the motion of the volume at a time to its motion at the next time.

\section{RESULTS}

In this part, an example of using the simulator is shown.

\section{A. Input Parameters}

Table 1 shows the parameters used for this example.

TABLE I. INPUT PARAMETERS

\begin{tabular}{|l|l|}
\hline \multicolumn{1}{|c|}{ Parameters } & \multicolumn{1}{|c|}{ Values } \\
\hline The period of the ECG signal & 1 second \\
\hline The velocity of the scanner & 270 degree per second \\
\hline $\begin{array}{l}\text { The number of } 360^{\circ} \text { tours of the } \\
\text { scanner }\end{array}$ & 5 turns \\
\hline
\end{tabular}

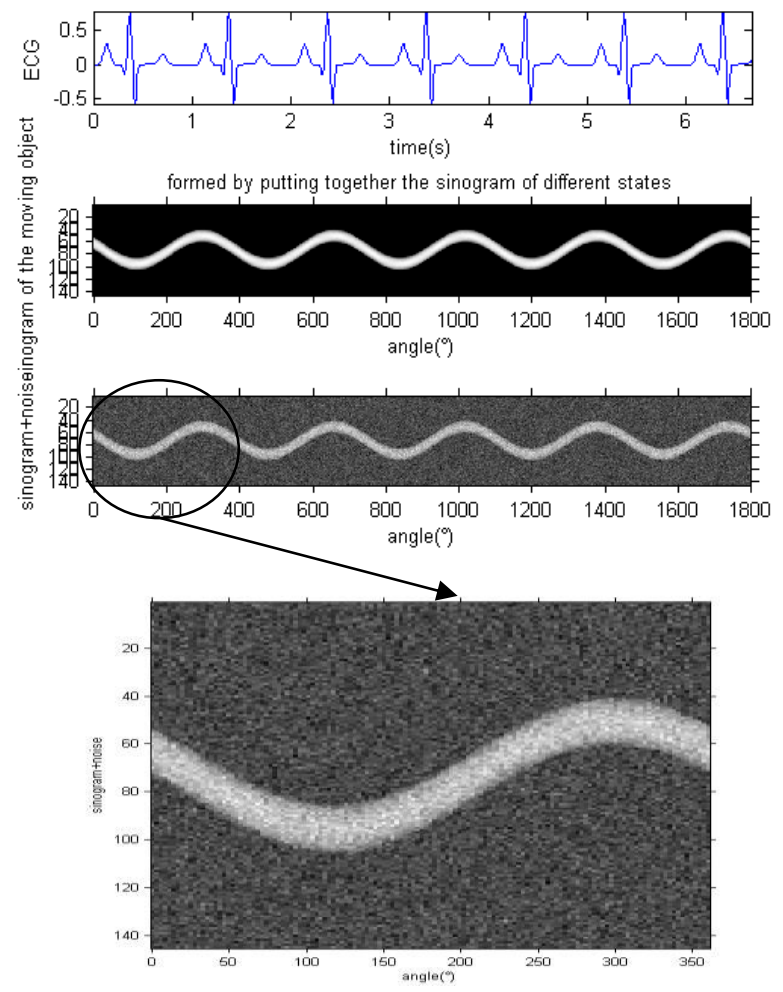

Figure 4. Outputs (The horizontal axes of the sinograms are the rotation angle and the vertical axes are the width of the X-ray foot print - the sinogram).

\section{B. Outputs} [1]

Fig. 4 shows the outputs for the input parameters of table

The first row shows the cardiac signal during the acquisition, according to which the modulation of fig. 2 is performed. The second row is the sinogram of the moving 
object made as explained previously. We also implemented the windowing which helps to retrieve the sinogram at a special time instant, as done in the process of four dimensional gated CT scans. In the last row, we added random noise to the sinogram to simulate the noise at the detectors, in the real process $\left(4^{\text {th }}\right.$ row: complete sinogram, $5^{\text {th }}$ row, zoom on the first $360^{\circ}$ or rotation). This sinogram now corresponds to the realistic simulation of the raw data of a CT scan machine from a moving object of arbitrary motion model.

An example of the reconstructed image from the realistic noisy sinogram is shown in fig. 5. The first image is the reconstructed image from the down-sampled noisy sinogram. This means that the number of the detectors is down-sampled to a realistic number in CT machines, as a source of error. The second image is the reconstructed image from all the sinograms with all the motion information. As expected, the borders are smooth due to the motion .The last image is the reconstructed image from the down-sampled sinogram with all the motion information.

a)
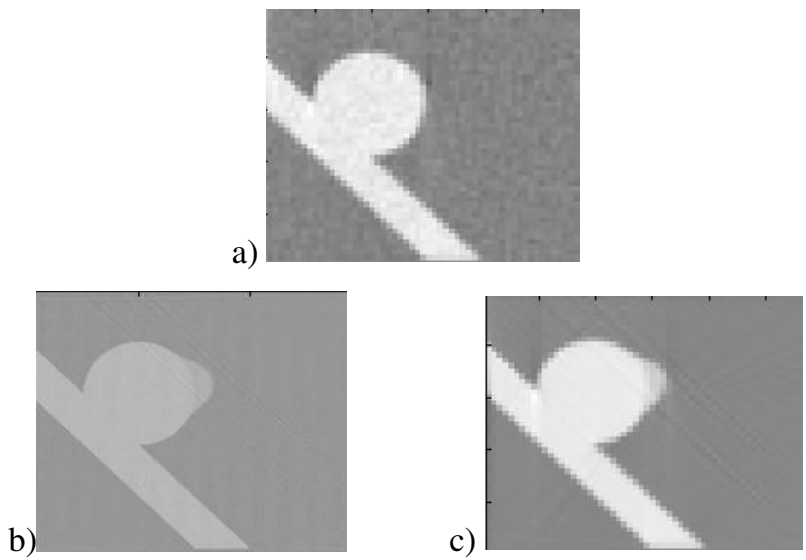

Figure 5. Reconstructed images: a) from the noisy down-sampled sinogram, b) from the sinogram with all the motion information, and c) from the down-sampled sinogram with all the motion information

\section{CONCLUSION}

We developed a realistic simulator to produce the raw data of the CT imaging of a moving object considering different criteria of the real acquisition. This digital phantom can be used by the medical image processing community not only to facilitate the testing before going to real phantoms or real data, but also serves as a tool to show and quantify the accuracy of different algorithms by a comparison of the result of any method with the a priori known ground-truth analytical model.

A first application of our work was used in [13]. It can also serve as an analytical precise ground truth to test different motion detection or motion correction algorithms, such as those used to detect or to correct pulmonary or cardiac motions.

\section{V.ACKNOWLEDGMENT}

This work was done in the frame work of the THROMBUS, a Quantitative Model of the Thrombosis in Intracranial Aneurysm, with the financial support of the European Commission, FP7 project.

\section{REFERENCES}

[1] Kevin M. Rosenberg, M.D, "CTSim: The Open Source Computed Tomography Simulator," Software, 2006, www.ctsim.org.

[2] X. Tang, and G. Wang, "Computed Tomography Simulator," in Encyclopedia of Medical Devices and Instrumentation, J. G. Webster, Ed. New Jersey: John Wiley \& Sons, Inc., 2006, pp. 266-277.

[3] C. A. Perez, J. A. Purdy, W. Harms, R. Gerber, J. Matthews, P. W. Grigsby, M. L. Graham, B. Emami, H. K. Lee, J. M. Michalski, Susan Baker, "Design of a fully integrated three-dimensional computed tomography simulator and preliminary clinical evaluation," Intl. J. of Radiation Oncology*Biology*Physics, vol. 30, no. 4, pp. 887-897, 15 Nov. 1994.

[4] D., O., Wiebers, J., P., Whisnant, J. 3rd, Huston, I., Meissner, R., D. Jr., Brown, D., G., Piepgras, G., S., Forbes, K., Thielen, D., Nichols, W., M., O'Fallon, J., Peacock, L., Jaeger, N., F., Kassell, G., L., Kongable-Beckman, and J., C., Torner, "International Study of Unruptured Intracranial Aneurysms Investigators. Unruptured intracranial aneurysms: natural history, clinical outcome, and risks of surgical and endovascular treatment," Lancet, 362, pp 103-110, 2003.

[5] Y., Ohashi, T., Horikoshi, M., Sugita, T., Yagishita, and H., Nukui, "Size of cerebral aneurysms and related factors in patients with subarachnoid hemorrhage," Surg. Neurol., vol. 61, no. 3, pp 239-45, discussion pp 245-7, 2004.

[6] F. Meyer, J. H. III, and S. Riederer, "Pulsatile increases in aneurysm size determined by cine phase-contrast MR angiography," J. Neurosurg., vol. 78, no. 6, pp. 879-883, 1993.

[7] J. Wardlaw, J. Cannon, P. Statham, and R. Price, "Does the size of intracranial aneurysms change with intracranial pressure? Observations based on color "power" transcranial Doppler ultrasound,” J. Neurosurg., vol. 88, no. 5, pp. 846-850, 1998.

[8] J. Wardlaw and J. Cannon, "Color transcranial "power" Doppler ultrasound of intracranial aneurysms," J. Neurosurg., vol. 84, no. 3, pp. 459-461, 1996.

[9] M. Hayakawa, K. Katada, H. Anno, S. Imizu, J. Hayashi, K. Irie, M. Negoro, Y. Kato, T. Kanno, and H. Sano, "CT angiography with electrocardiographically gated reconstruction for visualizing pulsation of intracranial aneurysms: Identification of aneurysmal protuberance presumably associated with wall thinning," Am. J. Neuroradiol., vol. 26, pp. 1366-1369, 2005.

[10] F. Ishida, H. Ogawa, T. Simizu, T. Kojima, and W. Taki, "Visualizing the dynamics of cerebral aneurysms with four-dimensional computed tomographic angiography," Neurosurgery, vol. 57, no. 3, pp. 460-471, 2005.

[11] Y. Kato, M. Hayakawa, H. Sano, M. Sunil, S. Imizu, M. Yoneda, S. Watanabe, M. Abe, and T. Kanno, "Prediction of impending rupture in aneurysms using 4D-CTA: Histopathological verification of a realtime minimally invasive tool in unruptured aneurysms," Minim. Invasive Neurosurg., vol. 47, pp. 131-135, 2004.

[12] Sh. Sepehri, and J-Ph. Thiran, "Direct Detection of Small Motion from Computed Tomography Images," Swiss Federal Institute of Technology, private communication, July 2012 (available at www.infoscience.epfl.ch ).

[13] Sh. Sepehri, and J-Ph. Thiran, "Direct detection of small motion from dynamic Computed Tomography images," $21^{\text {st }}$ European Signal Processing Conference, 2013, submitted for publication.

[14] Sh. Sepehri, Research Proposal for the Doctoral School in Electrical Engineering, Swiss Federal Institute of Technology, private communication, March 2012 (available at www.infoscience.epfl.ch )..

[15] C., Zhang, M.C., Villa-Uriol, M., De Craene, J., Pozo, and A., Frangi, "Morphodynamic Analysis of Cerebral Aneurysm Pulsation From Time-Resolved Rotational Angiography," IEEE Trans. Med. Imag., vol. 28, no. 7, pp1105-1116, 2009.

[16] M. Unser, Image Processing I, EPFL private communication, 2008.

[17] S. W. Smith, "The scientist and engineer's guide to digital signal processing," 1st ed. California Technical Pub., 1997. 